\title{
Primary pleural lymphoma of T cell origin in a paediatric patient with a focus on radiological findings - a case report and review of literature
}

\author{
Amirreza Jahanshahi ${ }^{1 A, E}$, Amirataollah Hiradfar ${ }^{2 A, B, D}$, Armin Zarrintan ${ }^{3 B, E, F, G}$, \\ Mohammad Mirza-Aghazadeh-Attari ${ }^{4 B, E, F}$
}

'Department of Radiology, Imam Reza Hospital, Tabriz University of Medical Sciences, Tabriz, Iran 2Pediatric Health Research Center, Tabriz University of Medical Sciences, Tabriz, Iran

${ }^{3}$ Imam Reza Hospital, Tabriz University of Medical Sciences, Tabriz, Iran

${ }^{4}$ Aging Research Institute, Tabriz University of Medical Sciences, Tabriz, Iran

\section{Abstract}

Purpose: Pleural lymphoma is a medical condition characterised by shortness of breath and obscure chest pain, which may be a diagnostic challenge, especially when it occurs in children. Plain chest X-rays and computed tomography (CT) scan are the main imaging techniques and are the initial diagnostic methods utilised.

Case report: A four-year-old boy was admitted to the emergency ward with pain in the right thoracoabdominal region, which had persisted for two months. Physical examination revealed reduced respiratory sounds in the right chest, but with no other significant findings. The patient underwent chest X-ray and CT scan, which showed right sided pleural thickening coupled with a massive pleural effusion. The patient underwent pleural biopsy, and a diagnosis of T-cell primary pleural lymphoma was made. The patient underwent treatment with BFM-NHL and was followed for three years, during which the patient remained disease free.

Conclusions: Pleural lymphoma is a rare diagnosis especially in children and could have overlapping clinical manifestations with more common conditions. Imaging techniques are the main route of clinical work-up towards final diagnosis. Here, we present a rare paediatric patient with no significant past medical history, who underwent imaging and was diagnosed with T-cell primary pleural lymphoma, a very rare subtype of primary pleural lymphoma.

Key words: CT scan, lymphoma, X-ray, PET, PET-CT.

\section{Introduction}

Pleural lymphoma is a medical condition characterised by shortness of breath and obscure chest pain, which may be a diagnostic challenge, especially when it occurs in children. Plain chest X-ray and computed tomography (CT) scan are the main imaging techniques and are the initial diagnostic methods utilised.

\section{Case report}

A four-year-old boy was admitted to the emergency ward with persistent pain in the right thoracoabdominal region, which had been present for at least two months. The pa- tient had recently developed dyspnoea and was complaining of extreme sweating, which had started a week ago. The patient was normotensive, had a heart rate of $80 \mathrm{bpm}$, respiratory rate of 21 breaths per minute and was not febrile. Blood oxygen saturation was $92 \%$ without external respiratory support. The patient had no history of recent infections or any documented immune deficiency syndromes. The patient did not have a significant past family medical history, and he was not a passive smoker. On clinical examination, the patient was agitated and had decreased respiratory sounds on the right chest. Percussion of the right thorax was dull. The patient's weight was $15 \mathrm{~kg}$, down from $19 \mathrm{~kg}$ six months earlier. The patient was admitted to the general paediatrics ward and underwent diagnostic

Correspondence address:

Dr. Mohammad Mirza-Aghazadeh-Attari, Aging Research Institute, Faculty of Medicine, Tabriz University of Medical Sciences, Daneshgah Avenue, Tabriz, Iran,

e-mail:m.aghazadeh75@yahoo.com

Authors' contribution:

A Study design · B Data collection · C Statistical analysis · D Data interpretation · E Manuscript preparation · F Literature search · G Funds collection 

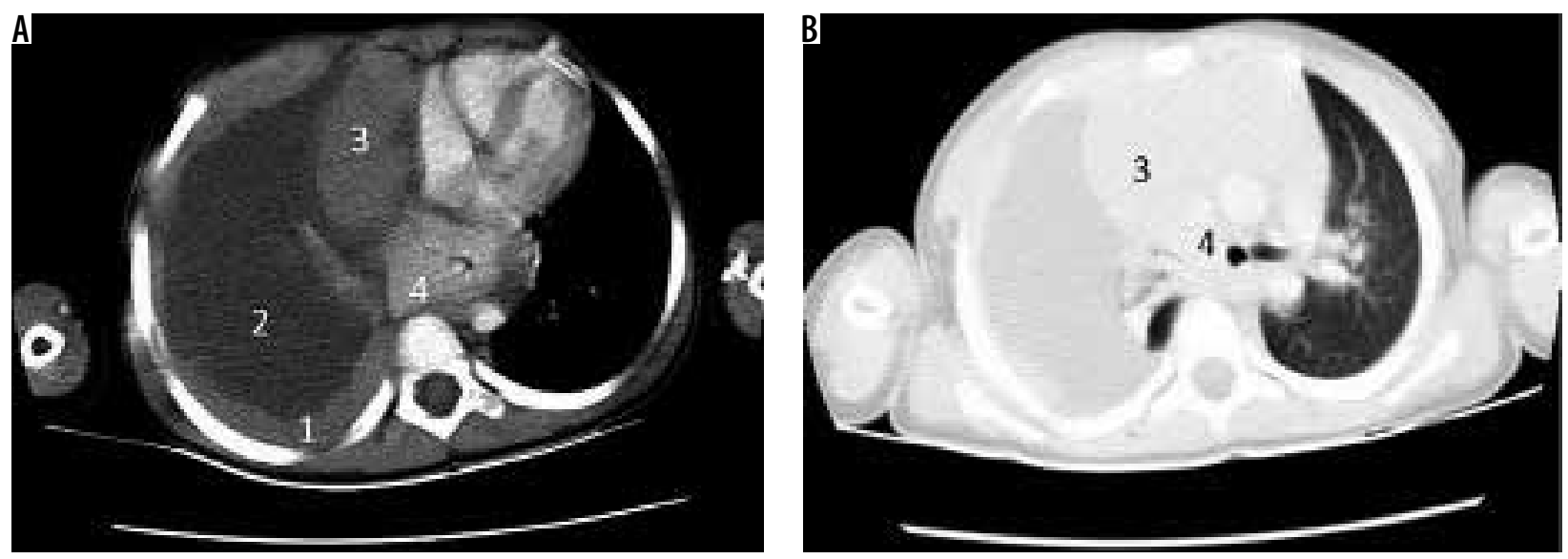

Figure 1. Initial computed tomography scan obtained from the patient. A) Mediastinal window, B) lung window. 1 - shows a diffuse right sided pleural thickening, 2 - shows a massive pleural effusion, 3 - shows the right lung which has collapsed because of the massive effusion, 4 - shows the shifting of the mediastinum to the other side

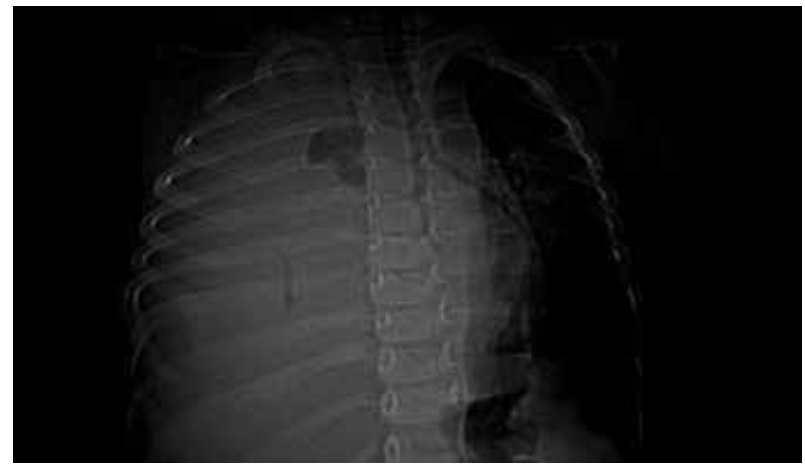

Figure 2. Scout view of the patient. Showing a massive effusion which has caused the mediastinum to shift to the left

imaging. Figures 1 and 2 illustrate the imaging results. Chest X-ray showed an opaque right thorax, with no other significant findings. CT scan revealed a nodular thickness of the pleura, with no mediastinal lymphadenopathy. After consultation from the surgical team, the patient underwent thoracotomy, and a biopsy was taken from the pleura. Macroscopic evaluation showed a cream tan mass with a heterogeneous cut surface. Microscopic evaluation showed a small cell malignant tumour, which consisted of monomorphic lymphoid cells with fine granular nuclear chromatin, with high mitotic rates and infiltration of the surrounding fat tissue and vascular structures with considerable necrosis. Immunohistochemistry resulted in the specimen staining positive for CD99, CD3, vimentin, and Ki67, while negative for CD20, CD34, CD79a, NSE, synaptophysin, and chromogranin. This was consistent with the diagnosis of acute lymphoblastic pre-T cell leukaemia. After the diagnosis was made, the patient received the BFMNHL treatment protocol, which consisted of two months of induction protocol, 50 days of consolidation protocol, 50 days of re-induction protocol, and finally 24 months of maintenance therapy [1]. Figure 3 illustrates the CT results after treatment. The patient was followed up for three years, and in this interval, periodic clinical examinations and PET-scans have shown that he remains disease free.
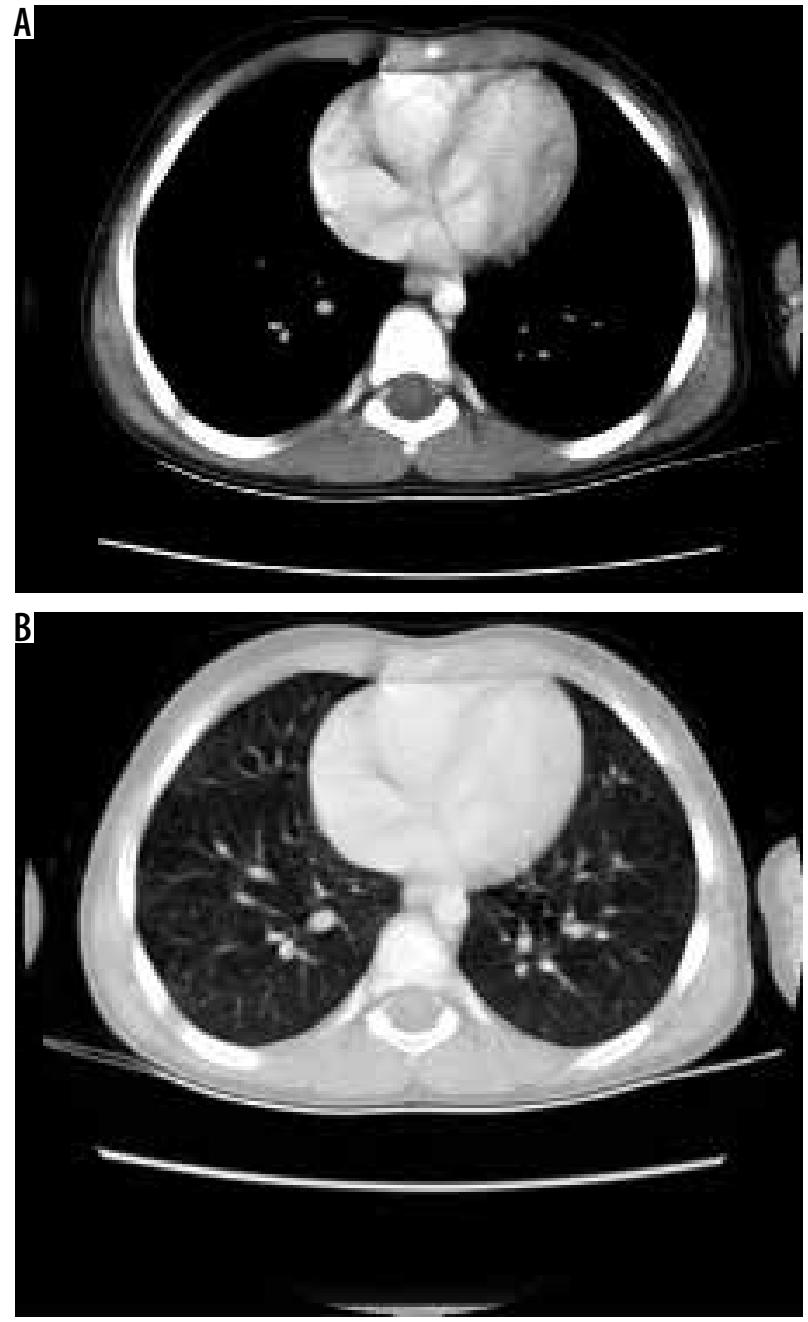

Figure 3. $A$ is the mediastinal window and $B$ is the lung window. Computed tomography scan obtained from the patient after the BFM-NHL protocol was administered. There were no signs of pleural effusion or pleural thickening. The shift in the mediastinum which was previously observed was absent

\section{Discussion}

Pleural lymphoma is a rare form of primary lymphoma, which presents with symptoms such as dyspnoea, pain on 


\begin{tabular}{|c|c|c|c|c|c|c|c|c|c|c|c|c|c|}
\hline 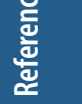 & $\simeq$ & 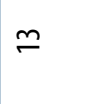 & 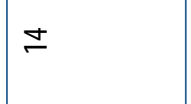 & $\stackrel{n}{n}$ & 0 & 0 & 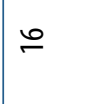 & $F$ & $\stackrel{\infty}{\perp}$ & 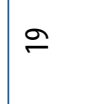 & $a$ & ㄱ. & in \\
\hline 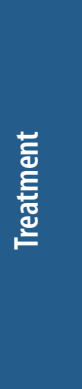 & 훙 & 훈 & $\stackrel{\Sigma}{\Sigma}$ & 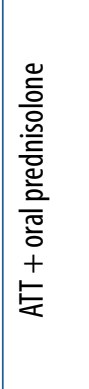 & $\stackrel{\Sigma}{z}$ & $\stackrel{\Sigma}{z}$ & 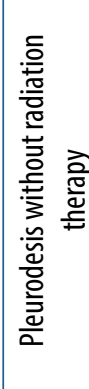 & 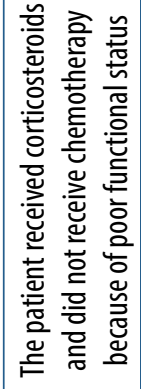 & 훔 & 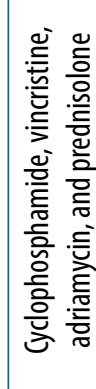 & 훔 & $\stackrel{\Sigma}{z}$ & 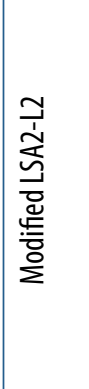 \\
\hline 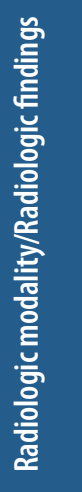 & 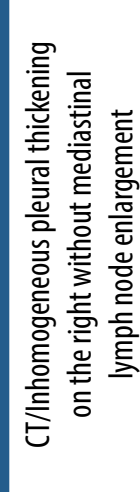 & 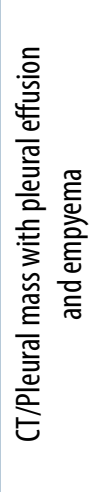 & 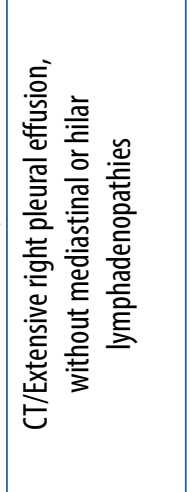 & 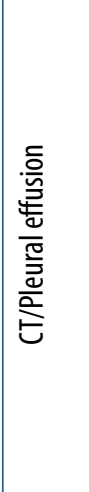 & 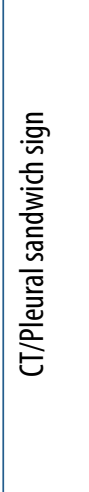 & & 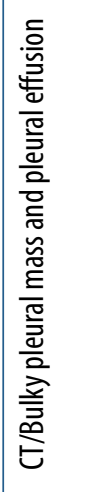 & 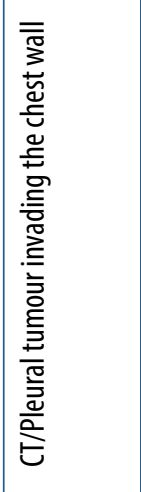 & 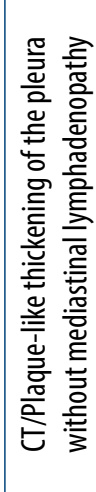 & 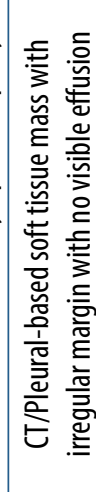 & 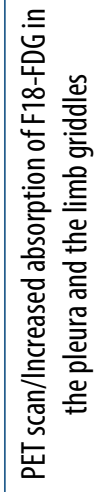 & 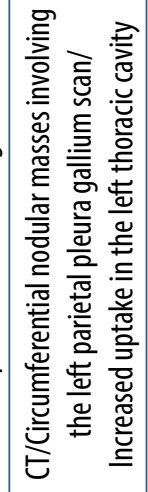 & 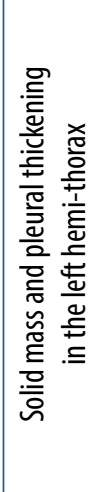 \\
\hline 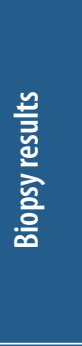 & 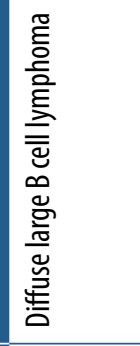 & 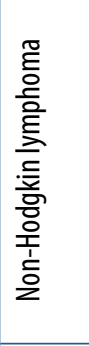 & 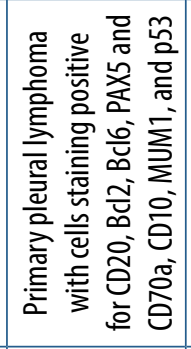 & 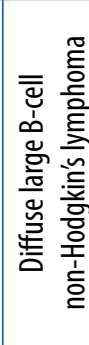 & 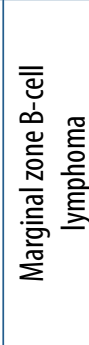 & 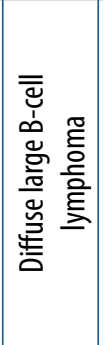 & 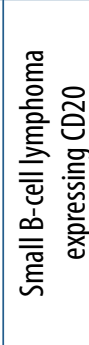 & 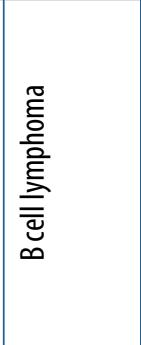 & 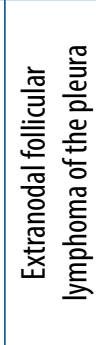 & 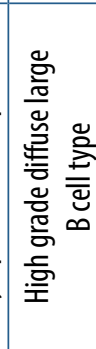 & 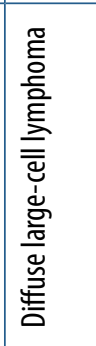 & 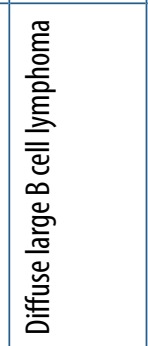 & 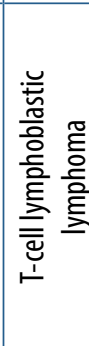 \\
\hline 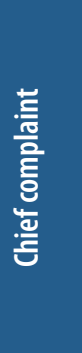 & 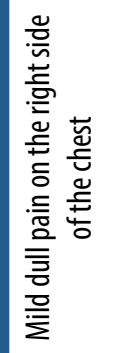 & 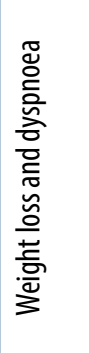 & 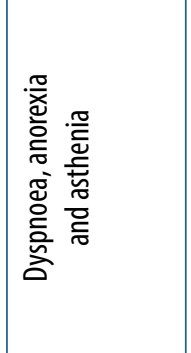 & 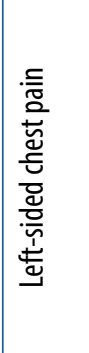 & 홍 & 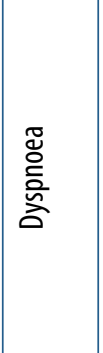 & 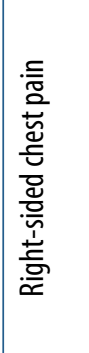 & 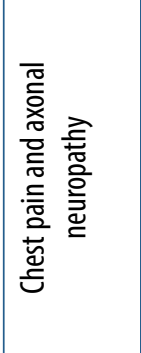 & 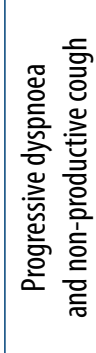 & 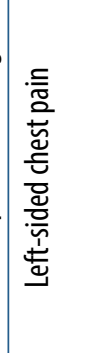 & 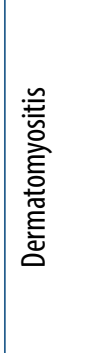 & 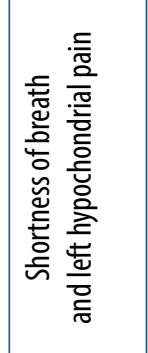 & 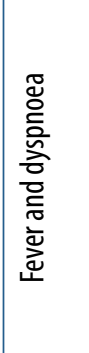 \\
\hline ๗ & 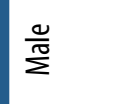 & $\frac{\stackrel{\Delta}{\pi}}{\sum \pi}$ & $\frac{\Delta}{\frac{\Delta}{\pi}}$ & $\frac{\Delta}{\sum^{\pi}}$ & 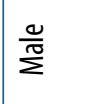 & $\frac{\Delta}{\frac{\Delta}{\pi}}$ & 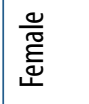 & $\frac{0}{\sum^{\pi}}$ & $\frac{0}{N^{\pi}}$ & $\frac{0}{\sum^{\pi}}$ & 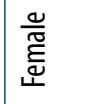 & $\frac{0}{\sum^{\pi}}$ & $\begin{array}{l}\frac{\mathscr{\Xi}}{\widetilde{\Xi}} \\
\text { एँ }\end{array}$ \\
\hline 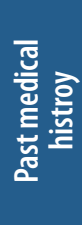 & 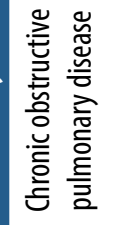 & 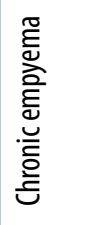 & $\stackrel{\Sigma}{z}$ & $\stackrel{\Sigma}{z}$ & $\stackrel{x}{z}$ & $\stackrel{\xi}{z}$ & $\stackrel{\Sigma}{z}$ & 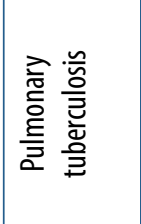 & $\stackrel{\Sigma}{z}$ & 离 & $\stackrel{s}{\Sigma}$ & $\stackrel{s}{z}$ & $\stackrel{\Sigma}{\Sigma}$ \\
\hline 푼 & 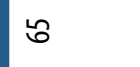 & $\infty$ & வ & $\stackrel{\infty}{\infty}$ & ஓ & 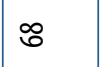 & N & $\approx$ & $\theta$ & 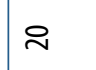 & 20 & $\stackrel{\infty}{\circ}$ & $\stackrel{\sim}{\sim}$ \\
\hline 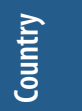 & 氠 & 高 & 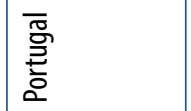 & 兽 & 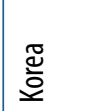 & 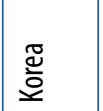 & 疍 & 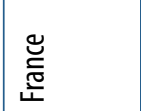 & 芯 & 営 & 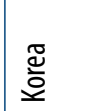 & 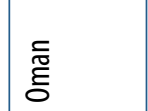 & 产 \\
\hline
\end{tabular}




\begin{tabular}{|c|c|c|c|c|c|c|c|c|c|c|c|c|c|c|}
\hline 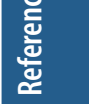 & $\bar{\sim}$ & $\approx$ & $\approx$ & 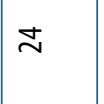 & \pm & $\nsim$ & $\stackrel{\sim}{\sim}$ & $\bar{\sim}$ & $\stackrel{\infty}{\sim}$ & $\stackrel{\infty}{\sim}$ & নి & 요 & $\bar{m}$ & $\bar{m}$ \\
\hline 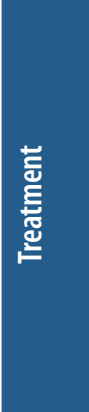 & 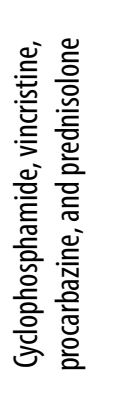 & 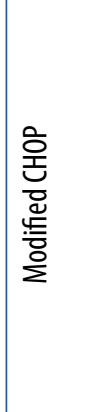 & $\frac{\Sigma}{z}$ & 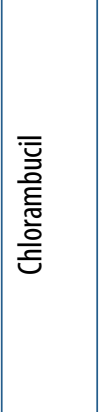 & 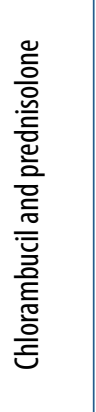 & $\stackrel{\xi}{z}$ & 훈 & 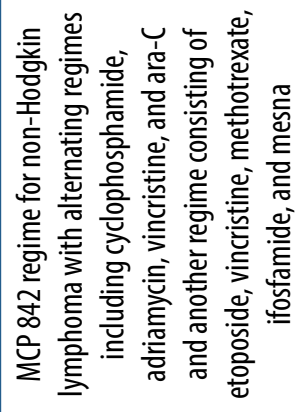 & 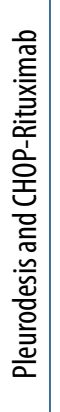 & 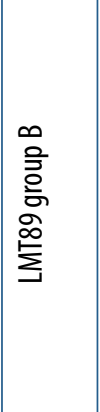 & $\frac{1}{z}$ & $\stackrel{\Sigma}{z}$ & $\stackrel{\Sigma}{z}$ & $\stackrel{\Sigma}{z}$ \\
\hline 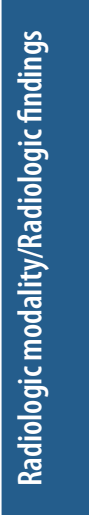 & 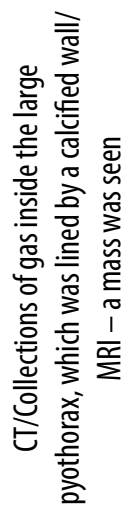 & $\frac{1}{2}$ & $\frac{\mathbf{z}}{\mathbf{z}}$ & 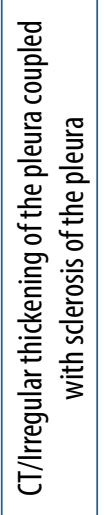 & 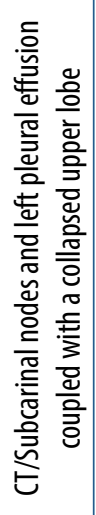 & 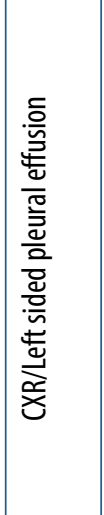 & 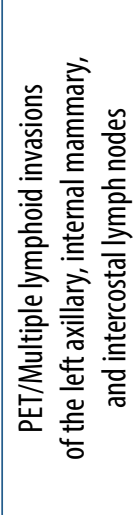 & 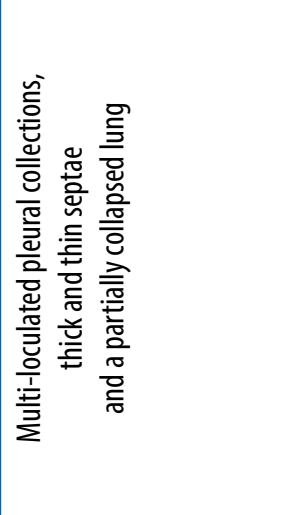 & $\stackrel{z}{z}$ & 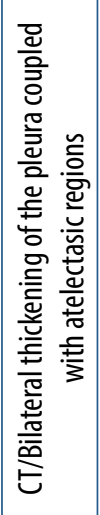 & $\frac{2}{2}$ & 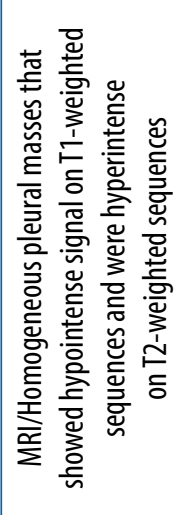 & $\frac{2}{2}$ & $\stackrel{\Sigma}{\Sigma}$ \\
\hline 善 & 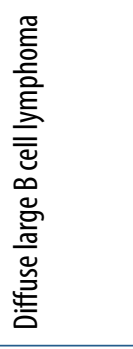 & 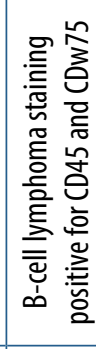 & 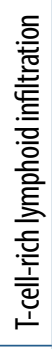 & 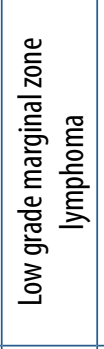 & 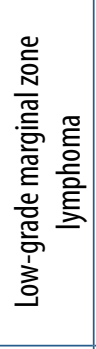 & 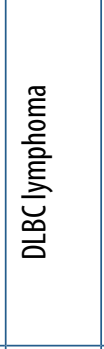 & 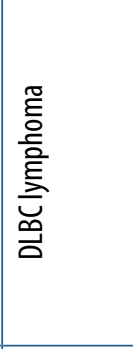 & 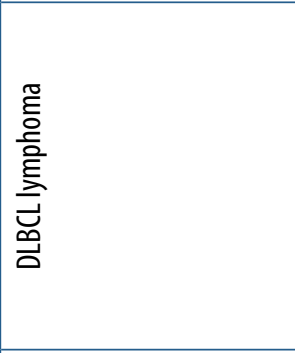 & 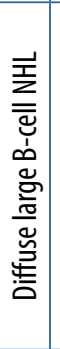 & 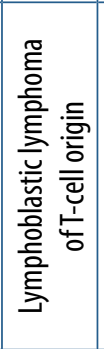 & 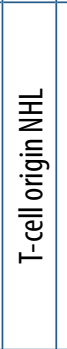 & 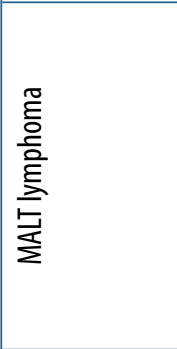 & 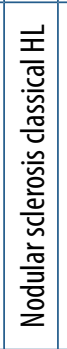 & 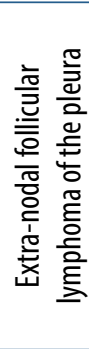 \\
\hline 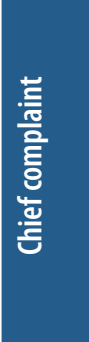 & 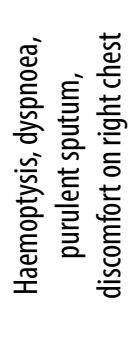 & 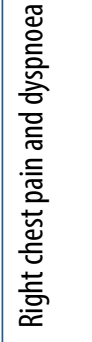 & $\stackrel{\Sigma}{z}$ & 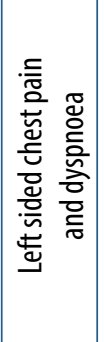 & 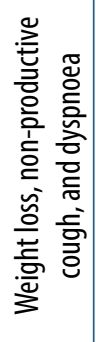 & 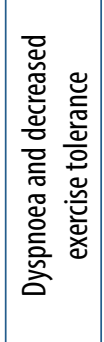 & 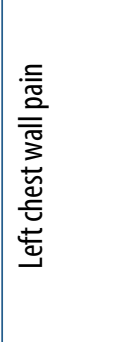 & 흠 & 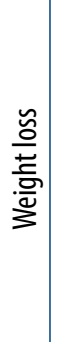 & 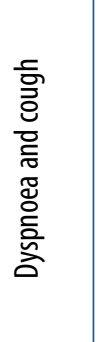 & 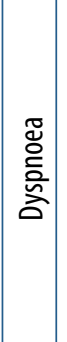 & 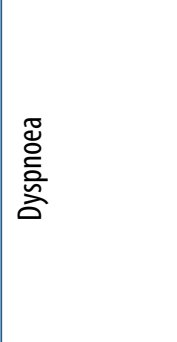 & 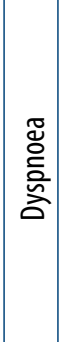 & 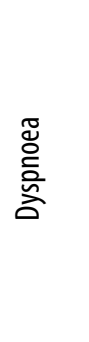 \\
\hline ๗ & $\frac{\frac{0}{\pi}}{\sum \frac{\pi}{\Sigma}}$ & $\frac{\frac{\Delta}{\pi}}{\sum}$ & $\stackrel{\Sigma}{z}$ & 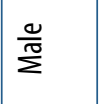 & $\frac{\Delta}{\frac{\Delta}{\pi}}$ & $\frac{\frac{0}{\pi}}{\sum}$ & 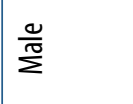 & 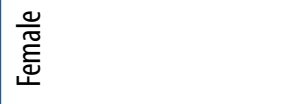 & 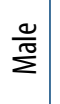 & $\frac{\Delta}{\sum^{\frac{\pi}{2}}}$ & $\frac{\frac{\Delta}{\pi}}{\frac{\pi}{2}}$ & $\frac{0}{\sum \pi}$ & 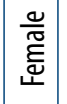 & 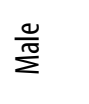 \\
\hline 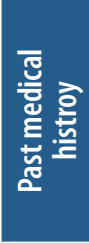 & 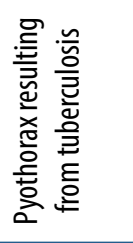 & 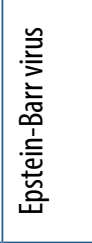 & 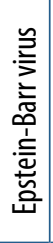 & 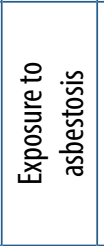 & 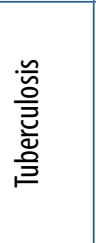 & 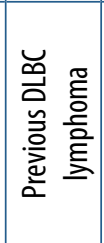 & $\stackrel{3}{z}$ & $\stackrel{\Sigma}{z}$ & $\stackrel{\Sigma}{z}$ & $\stackrel{\Sigma}{z}$ & 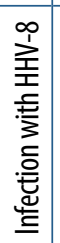 & $\stackrel{3}{\Sigma}$ & $\stackrel{\xi}{\Sigma}$ & $\begin{array}{l}\text { 岢 } \\
\text { 咅 }\end{array}$ \\
\hline ষ্ণ & $\kappa$ & $\Phi$ & $\stackrel{\mathbb{z}}{\mathbf{z}}$ & in & gr & $\pi$ & g̊ & $\simeq$ & $\vec{\sigma}$ & $\Xi$ & $\infty$ & $\approx$ & $\hat{m}$ & $\tilde{\theta}$ \\
\hline 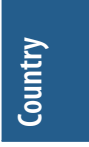 & 莺 & $\begin{array}{l}\text { 휴 } \\
\text { 흠 }\end{array}$ & $\begin{array}{l}\frac{\overline{0}}{\bar{a}} \\
\frac{1}{2}\end{array}$ & 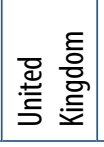 & 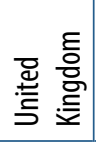 & 苞 & 胥 & 总 & 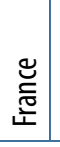 & 咅 & $\begin{array}{l}\text { 㫕 } \\
\text { 芯 }\end{array}$ & 言 & $\begin{array}{l}\stackrel{\Xi}{\Xi} \\
\stackrel{\Xi}{5}\end{array}$ & $\begin{array}{l}\stackrel{\Xi}{\Xi} \\
\stackrel{\Xi}{\Xi}\end{array}$ \\
\hline
\end{tabular}


the effected hemi-thorax, night sweats, decreased exercise capacity, and weight loss. Previous studies have shown that a considerable number of individuals with pleural lymphoma have risk factors such as a history of smoking, pulmonary tuberculosis, exposure to radiation, exposure to asbestosis, infection with Epstein-Barr virus (EBV), immunodeficiency, presence of chronic inflammation, and probably more [2,3]. Most cases reported are male and are usually over 40 years old. The diagnosis of primary lymphoma of the pleura is usually made by a combination of physical examination, imaging modalities, and biopsies. In physical examination, the patient may be without any significant findings or may present with decreased respiratory sounds. Percussion of the chest cavity may also illicit dullness on the effected hemi-thorax. After the initial examination, a combination of chest X-rays, CT scans, magnetic resonance images (MRIs), and positron emission tomography (PET)/CT scans may be used to investigate the pathology and to facilitate the act of obtaining a biopsy. Usually the biopsy shows an infiltrate of large B-cell lymphomas and marginal lymphomas. After the definite diagnosis, suitable treatment is initiated. The majority of cases presented to date have been treated with the CHOP regimen (cyclophosphamide, doxorubicin, vincristine, and prednisone), which is the standard treatment for nonHodgkin's B-cell lymphoma. This regimen has proven to be of beneficence for cases with diffuse large B-cell lymphomas, but has also shown considerable mortality, especially because of neutropaenic fever [4] (Table 1). In this case report, we discuss a four-year-old Azeri boy who was diagnosed with primary pleural lymphoma. The patient had no history of the aforementioned risk factors and was considerably younger than almost all patients previously reported with primary pleural lymphoma. Of note, the pathology of the lymphoma was of pre-T-cell lymphoma type, again a rare finding. In scientific literature, only a single similar case has been reported before. Karadeniz et al. reported a 2.5-year-old girl who was admitted to the Paediatric Hospital with the chief compliant of dyspnoea. CT scan showed a mass in the left thoracic cavity, and biopsy revealed that the malignant mass was indeed an infiltrate of lymphocytes positive for UCHL-1, a marker of T lymphocytes. The patient received the modified LSA2L2 therapy regimen and after the last cycle of chemotherapy, received 1800 cGy of cranial radiotherapy. After 11 months of remission the patient experienced an isolated relapse in the nervous system, which was treated with re-induction and more radiotherapy. The patient died four months after the relapse due to neutropaenic fever [5]. As the case presented in this report, the patient had no history of exposure to common risk factors and presented in a young age.

\section{Use of imaging modalities for pleural lymphoma}

\section{Chest X-rays and CT scans}

Usually, a chest X-ray is the initial diagnostic procedure undertaken. Pleural lymphoma could present with a ma- lignant pleural effusion, thickening of the pleura, and rarely a discrete nodule. The thickening of the pleura could be circumferential and present at the posterior aspect of the chest. Usually these findings are not accompanied by mediastinal lymphadenopathy. A rather specific finding observed in pleural lymphoma is the pleural sandwich sign, which is composed of a thin, highly enhanced central segment comprising mesenteric vessels, which is surrounded by enlarged pleural lymph nodes [6]. Although CT scans are considered the most helpful imaging technique in guiding the diagnosis of pleural lymphoma, the mentioned findings are not specific and other conditions such as bronchogenic cancers, metastasis, infections, chronic inflammatory processes, organising pneumonia, sarcoidosis, and lymphangitic carcinomas may have similar findings, making biopsy necessary for the diagnosis [7].

\section{PET/CT scan}

As mentioned, multiple benign lesions are listed as differential diagnosis of pleural lymphoma. PET scan is a suitable method to distinguish benign pleural lesions such as solitary fibrous lesions, lipomatous tumours, and adenomatoid tumours. Furthermore, it is an ideal tool to investigate individuals with no risk factor for pleural malignancies and cases with no obvious clinical diagnosis. Patil et al. reported the beneficence of PET/CT scan in detecting primary effusion lymphoma (PEL)-like lymphoma of the pericardium. PET scan revealed increased uptake in the pericardium, pleura, and the abdominal cavity, suggesting the presence of a primary pathology of the aforementioned locations and their serous linings [8]. Lee et al. reported another case in which PET scan was instrumental for the diagnosis. In this case report, PET scan was used to diagnose the relapse of a primary pleural lymphoma, which had manifested as a case of dermatomyositis [9]. PET scan was able to show the increased uptake of radiotracers in anatomical sites involved with dermatomyositis, and a nodular lesion with high uptake in the supraclavicular and retroperitoneal spaces.

\section{Ultrasonography}

Ultrasonography is the preferred method for the examination of pleural effusions because it is widely available (compared to CT-scans) and can detect small amounts of fluid in the pleural cavity. Ultrasonography is also a suitable method for the study of pleural lesions because it can detect masses in the pleura by their different echogenic characteristics. This imaging modality can also show signs of chronic inflammation in the pleura, such as fibrous bands and septa with encapsulated liquid, and is efficient in differentiating long-standing pleural effusion and thickening in pneumonia with malignant conditions such as primary lymphoma of the pleura or metastatic tumours. Ultrasonography may be useful in detecting lesions of the 
chest wall and differentiating them from lesions of the pleura. This is important because many chest wall lesions present with chronic pain in the thoracic region, a common finding in malignancies of the pleura [10].

Ultrasonography is also a suitable method for guiding biopsies of the pleura and can have important diagnostic implications in patients even after CT scans have been performed [11].

\section{Conclusions}

Pleural lymphoma is a rare presentation of lymphoma. This condition is usually seen in older men with risk factors such as smoking, previous tuberculosis infec- tion, and contact with asbestosis. In this case report we describe a child with pleural lymphoma, which was not associated with any risk factor. Pathological examination showed that the lymphoma was of T-cell origin, in contrast to most cases, which are of DLBCL origin. This patient was treated with the BFM-NHL protocol and remained disease free in three years of follow-up. Medical imaging techniques are the first line of any paraclinical examination series, and they guide further diagnostic and therapeutic actions.

\section{Conflict of interest}

The authors report no conflict of interest.

\section{References}

1. Reiter A, Schrappe M, Ludwig WD, et al. Intensive ALL-type therapy without local radiotherapy provides a $90 \%$ event-free survival for children with T-cell lymphoblastic lymphoma: a BFM group report. Blood 2000; 95: 416-421.

2. Martin A, Capron F, Liguory-Brunaud MD, et al. Epstein-Barr virus-associated primary malignant lymphomas of the pleural cavity occurring in longstanding pleural chronic inflammation. Hum Pathol 1994; 25: 1314-1318.

3. Molinie V, Pouchot J, Navratil E, et al. Primary Epstein-Barr virus-related non-Hodgkin's lymphoma of the pleural cavity following long-standing tuberculous empyema. Arch Pathol Lab Med 1996; 120: 288-291.

4. Cai P, Hao J, Wang D, Xu J. Comparative efficacy of different chemotherapies for non-Hodgkin lymphoma: a network-meta analysis. Oncotarget 2017; 8: 91238-91247.

5. Karadeniz C, Güven MA, Ruacan Ş, et al. Primary pleural lymphoma: an unusual presentation of childhood non-Hodgkin lymphoma. Pediatr Hematol Oncol 2000; 17: 695-699.

6. Kim Y, Lee M, Ryu YJ, Cho MS. The pleural sandwich sign in two cases of primary pleural lymphoma. Korean J Radiol 2015; 16: 213-216.

7. Bligh MP, Borgaonkar JN, Burrell SC, et al. Spectrum of CT findings in thoracic extranodal non-Hodgkin lymphoma. Radiographics 2017; 37: 439-461.

8. Patil VV, Sideras P, Machac J. PET/CT presentation of primary effusion lymphoma-like lymphoma unrelated to human herpes virus 8 , a rare NHL subtype. Indian J Nucl Med 2014; 29: 182-184.

9. Lee SM, Kim YK, Lee JJ, et al. (18)F-FDG PET demonstration of cancer recurrence presenting as dermatomyositis in a rare case of primary pleural lymphoma. Nucl Med Mol Imaging 2011; 45: 79-82.

10. Sperandeo M, Filabozzi P, Varriale A, et al. Role of thoracic ultrasound in the assessment of pleural and pulmonary diseases. J Ultrasound 2008; 11: 39-46.

11. Hallifax RJ, Corcoran JP, Ahmed A, et al. Physician-based ultrasound-guided biopsy for diagnosing pleural disease. Chest 2014; 146: 1001-1006.

12. Shao C, Guo Y, Xu X, et al. Non-pyothorax-associated primary pleural lymphoma without pleural effusion in an immunocompe- tent patient: a case report and literature review. J Thorac Dis 2018; 10: E368-E371.

13. Cerezo-Hernández A, Garcia-Gallardo Sanz MV, Arroyo Domingo CA, Del Campo Matias F. Pleural lymphoma associated with chronic empyema. Arch Bronconeumol 2018; doi: 10.1016/j.arbres.2017.12.014. [Epub ahead of print].

14. Fernandes FR, Oliveira RC, Rodrigues LV. Primary pleural lymphoma. Arch Bronconeumol 2018; 54: 430.

15. Baburaj P, Shankara B, George EJ, Gopinathan V, Prabhu D. Pleural lymphoma presenting as chylothorax. Journal of the Association of Physicians of India 2016; 64: 84-85.

16. Ru X, Ge M, Li L, Lin Y, Liu L. Primary pleural lymphoma: a rare case and a synopsis of the literature. J Thorac Dis 2013; 5: E121-E123.

17. Fajraoui N, Ben Hamida K, Hadj Kacem A, et al. Axonal neuropathy revealing pleural lymphoma as a long-term outcome of therapeutic pneumothorax. Rev Med Interne 2010; 31: e3-6.

18. Oikonomou A, Giatromanolaki A, Margaritis D, et al. Primary pleural lymphoma: plaque-like thickening of the pleura. Japan J Radiol 2010; 28: 62-65.

19. Basuthakur S, Sarkar A, Burman S, Dandale R. A rare case of pleural lymphoma. Lung India 2008; 25: 160-162.

20. Alsaadi K, Al-Dhuhli H, Hussain S, Burney I. Fused CT/Ga-67 SPECT images for detection of pleural lymphoma. Sultan Qaboos Univ Med J 2007; 7: 261-262.

21. Lee J, Yew WW, Wong PC, Fu KH. Non-Hodgkin's pleural lymphoma in long-standing tuberculous pyothorax mimicking suppuration. Int J Infect Dis 2001; 5: 167-169.

22. Danbara M, Takano Y, Fujino Y, et al. Development of pyothorax-associated pleural lymphoma in relation to focal cytokinemic condition and Epstein-Barr virus infection. Acta Haematol 1998; 99: 41-44.

23. Ibuka T, Fukayama M, Hayashi Y, et al. Pyothorax-associated pleural lymphoma. A case evolving from T-cell-rich lymphoid infiltration to overt B-cell lymphoma in association with Epstein-Barr virus. Cancer 1994; 73: 738-744.

24. Ahmad H, Pawade J, Falk S, Morgan J, Balacumaraswami L. Primary pleural lymphomas. Thorax 2003; 58: 908-909. 
25. Choy CF, Lee S. Pleural lymphoma. J Bronchol Interventional Pulmonol 2016; 23: 146-148.

26. Yang X, Xu X, Song B, et al. Misdiagnosis of primary pleural DLBCL as tuberculosis: A case report and literature review. Mol Clin Oncol 2018; 8: 729-732.

27. Ravikumar G, Tirumalae R, Das K. Primary pleural non-Hodgkin lymphoma in a child - an exceedingly rare disease. J Pediatr Surg 2012; 47: e29-e31.

28. Akbayram S, Dogan M, Akgun C, et al. Report of a non-Hodgkin lymphoma case presenting with pleural effusion. J Pediatr Hematol Oncol 2011; 33: e192-e194.
29. Lechapt-Zalcman E, Challine D, Delfau-Larue MH, et al. Association of primary pleural effusion lymphoma of T-Cell origin and human herpesvirus 8 in a human immunodeficiency virusseronegative man. Arch Pathol Lab Med 2001; 125: 1246-1248.

30. Barahona ML, Dueñas VP, Sánchez MT, Plaza BV. Primary mucosaassociated lymphoid tissue lymphoma as a pleural mass. Br J Radiol 2011; 84: e229-e31.

31. Steiropoulos P, Kouliatsis G, Karpathiou G, et al. Rare cases of primary pleural Hodgkin and non-Hodgkin lymphomas. Respiration 2009; 77: 459-463. 